\title{
Medicine and Money
}

\section{Everything you ever wanted to know about your anatomy}

Hold on to your clothes and run for cover. Whole body scanners are appearing on vacated land as rapidly as Starbucks. Diagnostic body imaging scans are becoming faster, better, and cheaper.

USA Today recently ran a feature on its cover, "The Inside Story" by reporter Robert Davis, that discussed a 20-minute whole body scan for about $\$ 700$. Davis had the examination surfside in Newport Beach, California, and the radiologists found "a bump" on his aorta that will apparently have to be monitored. Davis says he is glad he took the test.

Alternatively, you could go to "Inside Trac," in Beverly Hills, California. The 3 leading causes of death in America are heart disease, colon cancer, and lung cancer, so why not get all 3 organs scanned, just in case? An InsideTrac heart scan costs $\$ 375$. Add the body, and it becomes $\$ 795$, and for $\$ 1,325$, you also get the virtual colon study, which uses 3-dimensional reconstruction to exclude cancers and polyps larger than $6 \mathrm{~mm}$. You'll

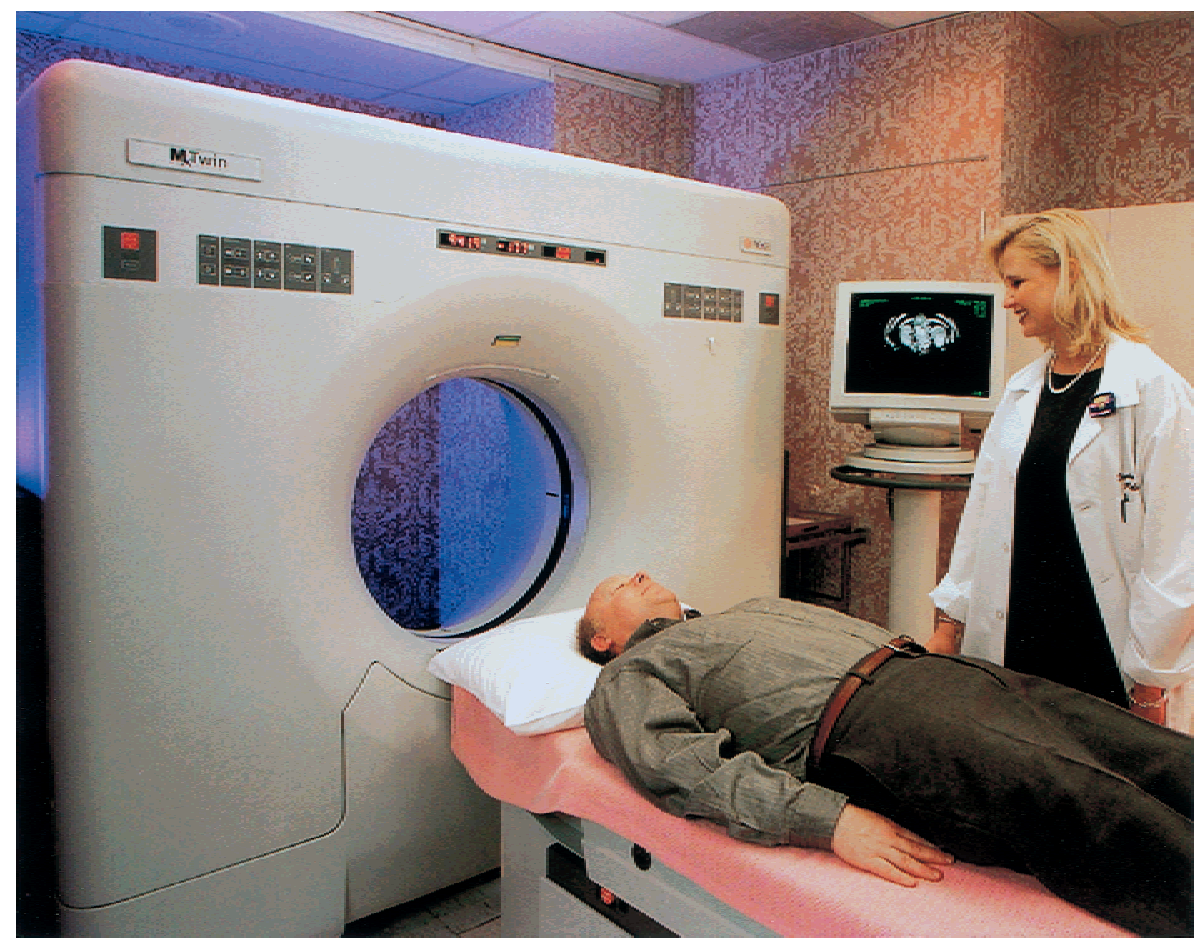

InsideTrac ${ }^{\mathrm{TM}}$ offers whole body scanning that is "painless, quick, and affordable"

need no anesthesia, laboratory work, or barium, and you won't feel a thing. The folks at InsideTrac say that they can scan you for early signs of disease in a mere 7 minutes.

Many in the medical establishment think that all this is unnecessary, expensive, and wasteful and that any little abnormality will lead to other more invasive, dangerous, uncomfortable, and costly tests. Perhaps they're right. Only time will tell. Our guess is that whole body scanning might someday be performed routinely.

Why not? The current costs of studying multiple anatomic areas are now less than what it originally cost to scan 1 site. Scanning costs are sure to come down even further. It is true that some findings will lead to more tests when indicated, just as in the past, but we can deal with that.

Why not do the tests sooner rather than later while the patient has a fighting chance? Some academic internists would rather make a late but exact diagnosis on the moribund than an early, less-than-perfect diagnosis on

374 wjm Volume 173 December 2000 someone who is clinically healthy. They often have this scientific principle that asserts that if they didn't have the idea first, invent the technology, do the first studies, or write the first articles, the concept simply doesn't cut the mustard. "Be neither the first to adopt the new," they say, "or to throw the old aside" unless, of course, it was their idea in the first place. But they have been wrong more than once.

The commonly held belief that men are more vulnerable than women to death by heart attack or colon cancer is incorrect. In addition, the rate of death of women from lung cancer is quickly approaching that of men. Thus, the scans offer something for everyone.

For the individual patient, the decision will not require complicated cost analyses. For the man or woman who is healthy, wealthy, wise, worried, or anxious, it will be a simple decision of "yes" or "no" to the scans. In fact, as more insurance companies pay for these screenings, their use will become more widespread. Perhaps, heaven forbid, such testing will actually eliminate other expensive laboratory and imaging modalities and reduce medical costs.

If these examinations can relieve anxiety, they could be worth the cost. As diagnostic radiologists, we like the idea of being primarily responsible directly to the patient. When abnormalities (or possible abnormalities) show up, we would refer patients to the appropriate physician or specialist. Perhaps this would be the start of a new trend in which radiologists would act as primary care physicians. We would certainly make sure that other physicians sent the patient back to us, the referring physicians. Let's call it vindication for the sins of the past when referring physicians took credit for our diagnoses.

In the managed care arena, these scans might appeal to the for-profit mass marketers, after they have done their inevitable marketing studies and focus group analyses. One week, for example, they might offer a special rate on a heart scan. The next week, it might

(374 wim Volime 173 Decenber 2000 
be a 2-for-1 and the next a 3-for-2 rate. Such great deals! Eventually someone will invent a handheld scanner like Doctor McCoy used in Star Trek. We propose calling it Inside Trac's StarTrec, the "real McCoy."

We know that this technology makes perfect sense on an intellectual basis, and we should probably have these scans done tomorrow. The problem is that we're not sure if we really want to know. But for most patients, this is a matter of personal choicewhich is the way it ought to be.

Authors Both authors are recovering diagnostic radiologists who retired just before the barium hit the fan. Michael Arnold Glueck, of Newport Beach (72143.2077@compuserve.com), has written extensively on medical, legal, mental health, and disability reform issues. Robert J Cihak, of Aberdeen, Washington (RCihak@techline.com), is president-elect of the Association of American Physicians and Surgeons.

Michael Arnold Glueck

Robert J Cihak

Competing interests: None declared

West J Med 2000;173:374-375

\section{A book to make you think}

Fevered Lives: Tuberculosis in American Culture since 1870 Katherine Ott, Harvard University Press, \$16.95, pp 296, ISBN 0 674 299116

In 1992, the New York Post ran the headline: "TB time bomb. Homeless contaminate public areas in city." This headline encapsulated much of the city's anxiety about the resurgence of the public health threat that is tuberculosis and the cultural forces that influenced the response. The recent epidemic in New York resulted in a reassessment of the role of public health officials, greater awareness of clinicians to drug-resistant strains and nosocomial spread within homeless shelters, prisons, and hospitals, and an increased public awareness of an ancient disease that many thought had been eradicated in the West. In the late 20th century, American society, and in particular New York society, re-evaluated its relationship with tuberculosis. And many of its responses were mirrored in the past.

In Fevered Lives, first published in 1996, Katherine Ott traces the cultural transformation of tuberculosis in America from 1870. She describes the changing "layers of meaning" that surrounded a diagnosis of tuberculosis and how, among the middle classes, this "most flattering of all diseases" of the 1870s was, as awareness of the social associations grew in the 1880s, transformed into a disease that was the consequence of either acquired or inherited degeneracy. She also describes how the disease mirrored "ethnic and racial fears and prejudices." For example, many believed that very different disease processes occurred in black people. "The seed of negro consumption is not in the lungs, stomach, liver or any organ of the body, but in the mind." By the turn of the century, however, the enthusiasm for blaming individual weaknesses was tempered by an increasing awareness that society's strictures were in part responsible.

Ott tells a fascinating story in describing the technology associated with medical advances, how these were interwoven with changing responses to and perceptions of disease, and how physicians' authority increased as they cornered the market in interventional expertise and microbiological understanding. At this time epidemiological awareness arising from "data-gathering techniques ... gave regularity and consistency to understanding of the disease," further contributing to the medical profession's authority. Despite its subtitle ("Tuberculosis in American Culture since 1870"), Ott's book largely concentrates on the period before the 1920s. Somewhat disappointingly, little is made of the advent of effective treatment and the more contemporary issues of drug resistance and the disease's associations with HIV. These issues, and the United States' response to them, have undoubtedly been influenced by past approaches.

In April 1993, increasing rates of tuberculosis led to the World Health Organization declaring a global emergency. As tuberculosis has enjoyed a resurgence, allied as it is to economic and social fractures and the HIV epidemic, so books on the disease are also enjoying something of a revival. The fact that several overlap in the period they cover, the issues they address, and their interpretation of their research findings is perhaps not surprising. Ott, however, offers much that is fresh. She argues persuasively that "the history of tuberculosis chronicles how a romantic, ambiguous affliction became first a dreaded and mighty social truncheon, and finally an entity bound up in the public health and civic order," and she uses powerful images to support her thesis.

Overall, Fevered Lives offers interesting insights into doctors', patients', and the public's changing understanding of, and response to, tuberculosis in the United States over a period of dramatic transition, and it describes well how different attitudes were reflected in the changing roles adopted by the different actors in the cultural tableau.

Competing interest: RJC's book From Chaos to Coercion: Detention and the Control of Tuberculosis was published by St Martin's Press in January 2000.

Richard J Coker

Imperial College School of Medicine at St Mary's Hospital, London 